LIBRO

Joaquín Fernández Abara, El Ibañismo (1937-1952):

Un Caso de Populismo en la Política Chilena

(Santiago: Instituto de Historia, Pontificia Universidad Católica de Chile, 2007).

\title{
JOAQUÍN FERNÁNDEZ ABARA, EL IBAÑISMO (1937-1952): UN CASO DE POPULISMO EN LA POLÍTICA CHILENA
}

\author{
Juan Luis Ossa
}

$\mathrm{U}_{\text {ne }}$ afirma que en Chile el movimiento "populista latinoamericano" de la primera mitad del siglo XX no estuvo ni siquiera cerca de manifestarse con la fuerza y el despliegue del populismo argentino, brasileño o mexicano. Acostumbrados a comprender este concepto bajo consideraciones peyorativas, o al menos un tanto despreciativas, hemos crecido oyendo sobre las características extraordinarias de la política chilena, en la que el orden imperante, el ejemplo del supuesto progreso decimonónico y el consenso mayoritario habrían impedido el auge de populistas emblemáticos, como Juan Domingo Perón, Getulio Vargas o Lázaro Cárdenas. A lo más, se sostiene que el caso de Carlos Ibáñez del Campo fue una excepcionalidad, una piedra pequeña en el camino casi perfecto de este lineamiento administrativo, antes que una práctica política racional y de largo alcance. ¿Cuán ciertas son estas aseveraciones?

Sin pretender insertar a su objeto de estudio en una discusión teórica sobre el populismo latinoamericano o europeo, el libro de Joaquín Fer-

Juan Luis Ossa Santa Cruz. D. Phil. (c), St Antony’s College, Universidad de Oxford. Licenciado en historia, Universidad Católica de Chile. 
nández, El Ibañismo: Un Caso de Populismo en la Política Chilena, viene, por un lado, a cuestionar las interpretaciones clásicas sobre el "populismo chileno", y, por otro, a complementar —y superar- las pocas iniciativas académicas que han estudiado el alcance de las adhesiones a Ibáñez entre los años 1937-1952. En cuanto al primer punto, al principio de la obra el autor entrega una definición de lo que generalmente se entiende por populismo: "el populismo puede ser definido como un movimiento político reunido en torno a un líder carismático, quien, identificándose con la totalidad del pueblo, pretende congregar grandes masas de adherentes” (p. 24). Además, dice Fernández, el populismo del siglo XX se manifestó preferentemente en sociedades "de masas en vías de desarrollo", en las que las "tradiciones y particularismos culturales de cada país" (expresiones de la cultura nacionalista, en palabras de Fernández) habrían sido estimuladas por hombres fuertes con el fin de integrar a la vida cívica a un alto porcentaje poblacional no identificado con los partidos políticos tradicionales.

A pesar de que, como bien plantea el historiador inglés Alan Knight ${ }^{1}$, trabajar el concepto "populismo" desde la perspectiva histórica puede ser una misión engorrosa cuando no superficial, entre otras cosas porque no es un fenómeno que se pueda cuantificar o medir, las definiciones de Fernández cumplen con su propósito, es decir, con insertar el papel político de Ibáñez dentro de las principales características del populismo. Con una mirada ante todo historiográfica (aun cuando cita, es cierto, a algunos autores provenientes de la ciencia política), su finalidad es utilizar el "populismo" como una herramienta heurística para explicar la "vasta complejidad de la historia" ${ }^{2}$ —en este caso, el contexto ibañista - antes que proponer una teoría. De ese modo, a lo largo del libro se encuentran un sinfín de actitudes protagonizadas por Ibáñez que pueden ser calificadas como "populistas". Sin embargo, estas calificaciones no son nunca monolíticas o modélicas, sino que se introducen dentro de la flexibilidad y dinamismo de un análisis eminentemente histórico.

En cuanto al segundo punto, y supliendo la falta de interés de los historiadores en constatar las características de esta — podríamos denominar- "corriente política” bajo la lente de la larga duración, la obra de Fernández es el primer intento serio en situar el ibañismo en todo su esplendor, y ya no simplemente como "un signo de la decadencia de otros actores políticos considerados como más importantes” (p. 21). Con ello, Fernández

${ }^{1}$ Knight, Alan: "Populism and Neo-populism in Latin America, Especially Mexico”, en Journal of Latin American Studies, Cambridge University Press, Vol. 30, No 2 (May, 1998), pp. 223-248.

2 Ibídem, p. 225. 
nos invita a leer una propuesta novedosa de un tema conocido por muchos, pero que, lamentablemente, ha sido postergado al rincón del olvido por la historiografía chilena de los últimos tiempos. No es que no existan estudios sobre Ibáñez; de hecho, existen, y muchos. Más bien, lo que el autor se ha propuesto es sobrepasar al personaje en cuestión, y rescatar las ideas y objetivos de quienes estuvieron detrás de su dilatada vida política. Así, este libro no es una biografía de Ibáñez, sino una radiografía de los múltiples personajes que, de una u otra forma, acudieron a su llamado, ya fuera para alentarlo o simplemente criticarlo.

El Ibañismo: Un Caso... es asimismo una prueba de la importancia que, en las últimas décadas, ha ido recobrando la crónica política en los anaqueles de la historiografía chilena y mundial. A la usanza de lo que Simon Schama propusiera en su alabado Ciudadanos. Crónica de la Revolución Francesa, Fernández recuperó una veta historiográfica rica en anécdotas, meticulosa en el análisis del día a día de las distintas coaliciones políticas y amena en el relato de los acontecimientos. No es de extrañar, pues, que en las páginas de esta obra encontremos una pormenorizada lista de las andanzas y devaneos de los actores involucrados en la época de Ibáñez. En ella, salen a la luz nombres conocidos por la gran mayoría: Arturo Alessandri Palma, Marmaduke Grove, Pedro Aguirre Cerda, Juan Antonio Ríos y Gabriel González Videla; aunque también otros menos patentes, como Jorge González Von Marées, Ricardo Latcham, Eduardo Cruz Coke y María de la Cruz. Todos ellos dan vida a esta suerte de "biografía colectiva" proyectada por Fernández.

Por otro lado, Fernández recoge en su libro una tradición historiográfica basada en el trabajo arduo y constante en los archivos públicos y privados, desafiando así a quienes han sostenido que la Historia —esa con mayúscula - se debe presentar a partir de compromisos ideológicos o de relatos en que supuestamente la interpretación debe vencer al documento. En ese sentido, El Ibañismo: Un Caso... es un ejemplo de la importancia de los hechos empíricos en la reconstrucción del pasado. Entre las fuentes utilizadas por Fernández cabría destacar los artículos de prensa, discursos públicos, cartas, caricaturas y proclamas partidistas publicadas en Chile entre los años 1930-1950. Todas ellas dan cuenta de un fenómeno del cual se ha hablado mucho, pero que, a mi entender, todavía no se ha trabajado en toda su extensión y relevancia. Nos referimos a la evolución de las capas medias chilenas en la primera mitad del siglo XX, y a la utilización que sus representantes hicieran de los medios escritos con el fin de sustentar sus aspiraciones. 
Es cierto, como bien lo demuestra Fernández, que diarios tradicionales como El Mercurio y El Diario Ilustrado hacían de voceros oficiosos de un mundo político ligado generalmente a las elites de “derecha”. Empero, no es menos cierto que muchas veces la clase media chilena encontró canales de participación en la propaganda escrita y que, más aun, el programa de los partidos tradicionales podía perfectamente asimilarse a los suyos. Así, la voz de los estudiantes universitarios, profesionales del Estado y miembros del ejército, por tan sólo nombrar a tres de los muchos sectores mesocráticos de esos años, nunca se apagó ni amilanó. Los artículos de las revistas Topaze, Ercilla y Hoy son prueba de ello.

Luego de estas disquisiciones historiográficas, veamos los planteamientos analíticos del autor. La tesis de Fernández, que cruza todo el libro y que abunda en detalles tomados de los archivos nacionales, señala que las tres elecciones presidenciales en las que participó Ibáñez (1938, 1942 y 1952) sirvieron de escenario para que amplios sectores de la población maduraran un culto en torno a la figura populista del General. En efecto, gracias a que apelaban, entre otras cosas, al "hombre común y sus valores”, al nacionalismo y a la exaltación del presidencialismo, los ibañistas se transformaron en "una importante tendencia populista en la política chilena" (p. 21), y como tales deben ser entendidos. A esto se debe la insistencia de Fernández de demostrar que, a pesar de su "laxitud y eclecticismo ideológico”, la propuesta de Ibáñez y su contribución a la política nacional generalmente se enmarcó dentro de una "versatilidad populista” (p. 24).

"Laxitud y eclecticismo ideológico" son, sin dudas, los conceptos que mejor describen al ibañismo. Entre sus filas no hubo grandes pensadores ni teóricos y, en general, los partidos políticos lo pensaron dos veces antes de sumarse a los proyectos de Ibáñez. A pesar de que, con el paso de los años, los ibañistas fueron afinando sus objetivos, el llamado a conformar y mantener una estructura dogmática sólida detrás del General nunca pasó de ser una quimera encabezada por unos pocos adherentes, los cuales, por lo demás, solían ser volátiles y acompañarlo únicamente en la actividad electoral. Las propuestas de Ibáñez, en tanto, también podían ser volátiles, cambiantes y flexibles. Esto explicaría su constante crítica a los partidos políticos, además del apoyo explícito de muchos grupos de independientes en todas sus incursiones presidenciales.

El afán de Ibáñez por desmarcarse de los partidos históricos, ya fueran de derecha o de izquierda, lo acercó al “pueblo”, cuya razón de ser se sostenía en la crítica a la democracia liberal defendida por los Partidos Conservador (PCC) y Liberal (PL). Sin embargo, lo anterior no fue óbice para que constantemente su pragmatismo lo llevara a solicitar el apoyo de los 
conglomerados tradicionales. Así ocurrió, por ejemplo, en los comicios de 1938, cuando infructuosamente intentó que el recién establecido Frente Popular (FP) — conformado por el Partido Radical (PR), el Partido Socialista (PS) y el Partido Comunista (PC), todos igual de "históricos" que el PCC o el PL- le entregara su apoyo electoral. Sólo las disputas internas entre la directiva del FP impidió que aquella alianza se concretara, obligando a que, al final de cuentas, el ibañismo fuera apoyado únicamente por agrupaciones menores —el Movimiento Nacional Socialista y la Unión Socialista, entre ellos—, aglutinados en la denominada Alianza Popular Libertadora (APL).

Ahora bien, restar importancia a este tipo de movimientos sería errado, entre otras cosas porque la APL se las arregló para erigirse entre la ciudadanía como "la unión de fuerzas que han de servir de base a una cruzada redentora” (p. 60). Tamaña afirmación, que recorrería no sólo la campaña de 1938 sino también las otras dos presentaciones electorales ibañistas, buscaba, a nuestro entender, un doble propósito: en primer lugar, aparecer ante el público como una alianza de hombres probos, ajenos a las prácticas más denostadas de la política nacional, como eran el cohecho y la supuesta malversación de fondos. En segundo, y muy ligado a lo anterior, plantear el ibañismo como una constante acción de rechazo al gobernante de turno (en este caso, Arturo Alessandri). De ese modo, Ibáñez alcanzaba un protagonismo distinto, sólo comparable al presidente que se disponía a dejar su mandato. La redención del país, entonces, iba de la mano de los ibañistas; su misión era alcanzar la total aniquilación de la corrupción de los políticos tradicionales.

Pero, como es sabido, no siempre el discurso y la práctica política corren por el mismo carril. En el caso de la elección de 1938, no fue el mesianismo de Ibáñez ni la "buena” campaña del FP lo que derrotó a los representantes de la democracia liberal, liderados por Gustavo Ross. Por el contrario, fue una coyuntura específica, con resultados concretos jamás intuidos y repercusiones inesperadas, lo que llevó a la tumba a la candidatura oficialista. Nos referimos a la Matanza del Seguro Obrero, y a cómo el desatino de las autoridades en la consumación del asesinato de cerca de sesenta jóvenes nacistas conllevó una completa re-configuración del mapa político nacional. A consecuencia de los eventos del 5 de septiembre, Ibáñez debió abandonar su candidatura, convencido, aunque a regañadientes, de que la única posibilidad de vencer a Ross era hacer un llamado a la unidad de la oposición y entregar su apoyo a Pedro Aguirre Cerda, representante del FP.

La unidad de la oposición conseguida a fines de 1938 no fue, sin embargo, de largo aliento. Como afirma Fernández, ya en mayo de 1939 
Ibáñez comenzó a alejarse de "la gestión gubernativa que estaba llevando adelante el frentismo". En un discurso radial "pronunciado por el General con motivo del Día del Trabajo" (p. 78), Ibáñez señaló que "la esperanza que el pueblo fijó en el Frente Popular se ha malogrado por la ambición y deslealtad de algunos hombres y dirigentes que forman parte de él. El programa que levantó la campaña presidencial del Excmo. Señor Aguirre Cerda permanece incumplido. [...] La administración pública es arrasada para dar cabida en ella a cuotas de partidarios” (p. 79). En menos de cinco meses, el ibañismo había llegado a la conclusión de que el gobierno del FP no conduciría a la tan esperada redención del pueblo chileno y que, a diferencia del General, Aguirre Cerda parecía lejos de encarnar los valores más "rescatables" de la historia nacional. Para muchos, Ibáñez representaba la continuación del régimen portaliano, reconocido por su vigorosidad y virtuosismo. Aguirre Cerda y su camarilla, en cambio, eran los forjadores de un "sistema que conduce a la irresponsabilidad en el mando, a la corrupción de los hábitos administrativos" (ibídem).

Entre 1939 y 1941, año de la inesperada muerte de Aguirre Cerda, Ibáñez acentuó sus críticas al gobierno, distanciándose no sólo del radicalismo sino también de otros movimientos de izquierda, como el PC. Obviamente, aquel distanciamiento redundó en una alianza cada vez más explícita con amplios sectores derechistas, los cuales vieron en Ibáñez al "hombre fuerte" que traería unidad y progreso. Con el objetivo de conformar una “Candidatura Nacional”, en diciembre de 1941 el diario El Mercurio sintetizó los objetivos que debía encarnar dicha candidatura: construir un gobierno "sin distinción de banderas y de banderías"; "emprender una labor en favor del orden de las finanzas”; “despolitizar la administración pública y los sindicatos"; y "repudiar las presiones de masas sobre los criterios de eficiencia en la labor administrativa, haciendo valer de esta manera el principio de “jerarquía”' (p. 90). Es decir, El Mercurio se sumaba a las críticas del ibañismo sobre el papel jugado por el FP en la política nacional. De ahí a conformar un gran bloque opositor entre el PCC, el PL y los seguidores de Ibáñez, había un solo paso.

Aunque no fue fácil dar ese paso - debido, entre otras cosas, a que tanto en el liberalismo como en el conservadurismo hubo quienes se mostraron reticentes a apoyar a un ex dictador (Ibáñez actuó como tal entre 1927-1931) — el acuerdo entre las derechas y el General quedó sellado entre los últimos días de 1941 y los primeros del año entrante. A ellos, se unieron muchos de los ibañistas de la elección de 1938 (incluidos ciertos partidos de centro-izquierda, como el Partido Radical Socialista) y, sobre todo, diversos grupos de independientes con claras tendencias nacionalistas. 
Uno de los puntos altos del libro de Fernández dice relación con el análisis de estos grupos nacionalistas. Continuando con la línea de investigación de historiadores como Verónica Valdivia Ortiz de Zárate y Markus Klein, el autor concluye que, durante el período del FP, "acudimos a un proceso de refascistización de los movimientos nacionalistas chilenos" (p. 109). La revista Acción Chilena fue la encargada de difundir estas "nuevas voces del nacionalismo chileno”. En uno de sus artículos, la revista entregó su apoyo al General, destacando su condición de militar e integridad moral:

Un hombre que sabe llevar la espada es muchas veces necesario para una nación; en ocasiones es imprescindible para salvarla... las fuerzas armadas, es preciso repetirlo, son el baluarte de nuestra integridad geográfica y de nuestra integridad moral. De nada sirve un país con sus fronteras intactas si tiene el alma destrozada o enferma. El General Ibáñez encarna la pureza de nuestras instituciones de defensa. Es, a no dudar, el candidato que mejor representa las bondades de nuestra raza (p. 110).

A las elucubraciones raciales y chauvinistas de Acción Chilena, se sumó la acción de otros movimientos fascistas e independientes. Sin duda, ambos sectores compartían la impresión de que, al haber sido miembro de las Fuerzas Armadas, Ibáñez daba garantías de independencia doctrinaria. Eso explicaría por qué, a lo largo de los años, el General recibió el apoyo constante de sus correligionarios de armas, quienes vieron en Ibáñez el epítome del político "no profesional” y el símbolo del anticomunismo.

Lo anterior no es un dato menor. Si en la elección de 1938 Ibáñez había representado la "alternativa populista antioligárquica de nacionalismo revolucionario” (p. 115), ahora, en 1942, se erigía como un nacionalista anticomunista cuyo objetivo era asegurar la "armonía entre patrones y obreros" (p. 114). En otras palabras, su discurso continuaba siendo más o menos populista —el cuarto punto de su programa prometía traer: "aumento de la producción, estabilización de precios y costo de la vida de acuerdo con la capacidad adquisitiva de la población”, pero ya no desde una vertiente izquierdista, sino cercana a los "partidos de derecha". Con el fin de congeniar el soporte de los grupos de independientes con el del PCC y el PL, los líderes de la campaña "evitaron profundizar las críticas a los partidos políticos”, resaltando, eso sí, que Ibáñez simbolizaba "los intereses de Chile, por sobre las clases y círculos” (p. 115). Por ello, no es de extrañar que El Mercurio, órgano semi oficial de la derecha chilena, insertara el 1 de enero 
de 1942 un afiche en el que se situaba al General en la línea de Diego Portales y José Manuel Balmaceda, dos personajes de la historia de Chile bastante disímiles entre sí pero defendidos tanto por independientes como por los partidos tradicionales. El afiche hacia alusión al "legado portaliano" y rezaba: "Portales, el Creador”; "Balmaceda, el Defensor”; "Ibáñez, el Restaurador” (p. 117).

La última gran característica de la campaña presidencial de 1942 dice relación con la masificación de la política nacional. Desde hace años que el debate electoral se había tomado el espacio público, dando pie a que la participación popular se manifestara a través de reuniones ideológicas o concentraciones masificadas. Así lo demostró la denominada "Marcha de la Victoria" que, según algunos, reunió a más de cien mil ibañistas el 4 de septiembre de $1938^{3}$, y cuyo recuerdo debió pesar cuando se iniciaron los preparativos del llamado "Desfile de la Patria", llevado a cabo el 28 de enero de 1942. En la ocasión, miles de adherentes oyeron atentos el discurso de clausura pronunciado por Ibáñez junto a la estatua del general Bulnes. En él, Ibáñez hizo un recuento de sus seguidores y dio señales de cómo sería su futuro gobierno:

Mi partido es hoy la Patria [en ella] Se reúnen las colectividades políticas más poderosas y de mejor organización; los grupos laboriosos de la agricultura, de la producción y del comercio; sectores no contaminados de las fuerzas de izquierda, que no han querido seguir los estandartes de una combinación responsable de actos que la historia sancionará enérgicamente... Llegaré a la primera magistratura sin compromisos con fracciones ni con individuos. Tendré sólo la sagrada obligación de formar un gobierno con los hombres más capaces de todos los sectores que me acompañan (p. 125).

Sin embargo, otra vez el discurso populista —ahora, insistimos, de corte nacionalista pero anti-revolucionario- de Ibáñez no dio resultado: el General perdió la elección por más de cincuenta mil votos, aunque logró vencer a Juan Antonio Ríos, candidato oficialista, en importantes sectores de Santiago y Valparaíso. Como concluye Fernández, "el importante potencial de movilización electoral de una estrategia populista quedó manifiesto en las elecciones de 1942" (p. 125).

El último tercio del El Ibañismo: Un Caso... da cuenta de la evolución de ese potencial electoral a lo largo de la década de 1940, culminando

${ }^{3}$ Zorrilla, Enrique: La Profecía Política de Vicente Huidobro (Santiago: Ediciones Nuestramérica, 1996), p. 120. 
con el análisis de la elección presidencial de 1952. De los tres capítulos que contiene el libro, este último es, sin dudas, el más acabado en términos analíticos y narrativos. Comienza con un certero estudio sobre el origen del Partido Agrario Laborista (PAL) y el papel jugado por sus líderes en la configuración del último gran referente ibañista.

La historia del PAL es simple: la Alianza Popular Libertadora, aquella congregación ibañista forjada en la elección de 1938, había logrado mantenerse más o menos viva durante los gobiernos radicales, a pesar de que su relevancia se había visto mermada por el auge de un sinnúmero de nuevas agrupaciones que, de una u otra forma, rodeaban al General. Una de éstas, el Partido Agrario, encabezado por Jaime Larraín, se había transformado en el "ente representativo de los productores agrícolas, logrando aglutinar en torno suyo a importantes sectores de sensibilidades corporativistas y rechazando a los partidos tradicionales” (p. 131). El problema del partido de Larraín era que, al igual que la APL, no tenía gran presencia electoral, cuestión que lo llevó a acercarse a ella y a crear un solo gran referente ibañista, conocido como agrariolaborismo ("el partido añadió el apelativo 'laborista' al nombre de la colectividad con el fin de mostrarse como una organización representativa de fuerzas productoras”) (p. 132).

La relación del agrariolaborismo con el gobierno radical de Gabriel González Videla fue de altos y bajos; de complementación, pero también de denuestos y críticas. La discusión en torno a la Ley de Defensa de la Democracia en 1948 conjugó a la perfección dicha relación. Por un lado, el PAL se mostró complacido con el quiebre entre González Videla y el comunismo; no obstante, por otro, se negó a aprobar "el artículo que borraba a los militantes del Partido Comunista de los registros electorales” (p. 134), generándose un ambiente de tensión entre el radicalismo y el nuevo partido agrariolaborista. Ibáñez, por su parte, cortó definitivamente con el oficialismo, e incluso estuvo involucrado en una intentona de golpe de Estado, conocida como el “Complot de las Patitas de Chancho". La participación del General en esta aventura, que buscaba terminar con el gobierno de los partidos e instalar a Ibáñez en el poder, fue una nueva señal de que el ibañismo estaba dispuesto a enfrentar cualquier circunstancia con tal de alcanzar la primera magistratura. La campaña presidencial de 1952 confirmaría esta aspiración.

La elección de 1952 consagró al populismo ibañista. De hecho, fue en estos comicios cuando el General aceptó, sin tapujos ni segundas lecturas, que se le asimilara a los populistas más reconocidos de la región, Juan Domingo Perón entre ellos (no deja de ser interesante que Ibáñez "aceptara" la candidatura presidencial "mientras se encontraba en territorio trasandino" (p. 187). Algunos miembros —no todos— del Partido Socialista 
Popular (PSP), cuyo origen se debió a una escisión del Partido Socialista luego de la discusión sobre la Ley de Defensa de la Democracia, fueron clave en el acercamiento de Ibáñez a los sectores populistas latinoamericanos. Como decíamos al principio, el objetivo de Fernández no fue insertar a su objeto de estudio en un marco global regionalista, pero, por lo menos en esta última parte del libro, existe un intento por situar a Ibáñez dentro de un marco referencial más amplio. Es así como encontramos ciertas declaraciones del PSP que permiten comprobar el acercamiento del General con los grupos populistas anteriormente nombrados.

De acuerdo con los socialistas populares, “América Latina vivía, hacia comienzos de los años 50, un auge de los 'movimientos nacionales y populares', representados entre otros por el Movimiento Nacional Revolucionario Boliviano y el peronismo argentino" (p. 156). La oportunidad de engendrar una verdadera "revolución latinoamericana" estaba al alcance de la mano, y a Ibáñez le cabía un importante rol en su consagración. Obviamente, algunos socialistas se oponían a que "el ibañismo, al igual que el peronismo, recurriera 'demagógicamente a las más absurdas medidas de corte dictatorial”' (pp. 156-157). Empero, al mismo tiempo, socialistas como Clodomiro Almeyda hacían denodados esfuerzos por convencer a sus correligionarios de que Ibáñez era "el único candidato capaz de concitar el suficiente apoyo popular para 'parar a la derecha”” (p. 157). Incluso más, Almeyda era de la opinión de que los socialistas chilenos no debían cometer el mismo error que sus compañeros argentinos, cuya dura oposición al peronismo terminó "por separarlos y antagonizarlos con la mayoría del pueblo" (ibídem).

La colaboración entre el ibañismo y el socialismo popular llegó a buen puerto en julio de 1951, aunque no tan fluidamente como Almeyda habría deseado. Salvador Allende, a la sazón senador y el más severo crítico del ibañismo al interior del PSP, declaró su animadversión por el General en una entrevista publicada por la revista Ercilla. Sus palabras, un tanto contradictorias con lo que él mismo haría años más adelante cuando decidió convocar a los militares a su gobierno, son, sin embargo, una primera señal de "esa curiosa mezcla de doctrinarismo marxista y cultura liberal que lo acompañaría por el resto de su carrera política” (p. 162):

Comprobé la decisión irrevocable de la mayoría de la directiva del PSP de llevar adelante la candidatura del 'Senador' Ibáñez. Y subrayo 'Senador'. Está bueno de terminar con lo de 'General'. Ibáñez dejó de ser militar cuando metió al ejército en funciones que no le correspondían (...) Hablaré del 'Senador’ Ibáñez, es un activo profesional de la política. De una 
actividad versátil, curiosa y paradojal. Abanderado de la derecha, líder popular de la última hora. [...] Los sectores que acompañan a Ibáñez son revoltosos y heterogéneos. Es tan imposible conciliar los intereses del latifundista agrario con los del campesino socialista, como los principios fascistas de los dirigentes ibañistas con el pensamiento socialista de nosotros. [...] En esta lucha presidencial están en juego conceptos sobre convivencia social y democrática que obligan a los hombres a definirse (pp. 161-162).

Y lo cierto es que Allende no demoraría en definirse, fusionándose rápidamente con el resto de los socialistas anti-ibañistas en el Movimiento de Recuperación Socialista. Como era de esperarse, Allende fue el candidato presidencial de dicho sector, dando pie a una dura respuesta de parte de socialistas populares como Óscar Waiss, quien, un año después de la elección presidencial, continuaba refiriéndose en forma despectiva a Allende: "La cara afeitada y pulcra del doctor Salvador Allende, pije, charlatán y siútico. Una cara que se parece muy poco a la del verdadero pueblo revolucionario" (p. 163).

En el análisis de la campaña de 1952 acudimos también a un resurgimiento de los movimientos independientes pro ibañistas. Fue, como dice Fernández, una verdadera "eclosión” de organizaciones no afiliadas a partidos tradicionales la que apoyó al candidato opositor, entre las cuales destacaban el Movimiento de Renovación Nacional y el Partido Femenino de Chile. El primero, a través de su diario La Escoba, dio origen al que fuera - y es - el mote más conocido en torno a Ibáñez, aquel que señalaba que el General "barrería” con la politiquería y la corrupción de los gobiernos radicales. El segundo, en tanto, aportó una nada insignificante cuota de votantes femeninas a las urnas ibañistas, y su líder, María de la Cruz, se transformó en un ejemplo del "nacionalismo antioligárquico, moralista, antipartidista” y autoritario del ibañismo de 1952. En esta misma línea, de la Cruz no dudó en asimilar a Ibáñez con Perón, declarando que “el peronismo es la realización de la cristiandad” y que aquél era "tan popular como el ibañismo en Chile” (p. 178).

Estas alusiones de María de la Cruz dan cuenta del marcado tinte peronista y autoritario que fue tomando la campaña de Ibáñez. Sindicado como el "General de la Esperanza”, Ibáñez trazó su futuro gobierno como una continuación de su primer gobierno, cuyas características dictatoriales lejos de amedrentar satisficieron a la mayoría de la ciudadanía. Que Ibáñez movilizara "a un electorado pluriclasista, obteniendo altísimas votaciones en sectores de clase baja y clase media y un importante respaldo en secto- 
res de clase alta en las zonas urbanas" es una prueba contundente de lo anterior. También lo es el hecho de que Ibáñez concitara la adhesión de votantes apáticos, “aumentando a más de un $85 \%$ el porcentaje de votación de los ciudadanos inscritos, de los que en la elección anterior sólo había participado un 75,9\%” (p. 190). Gracias a este aumento electoral, el General pudo por fin llegar al poder mediante el voto popular, demostrando que "el llamado a los independientes y la interpelación a las masas mediante un discurso nacionalista habían dado resultado" (ibídem).

Decíamos al principio que este libro no es una biografía de Ibáñez, sino más bien una radiografía de la participación política de sus adherentes en un período determinado, que culmina en 1952. Esto no impide, por supuesto, que al lector le surjan interrogantes sobre qué sucedió con el ibañismo después de esa fecha, como también acerca del papel del ibañismo en la historia de Chile y si acaso ese papel puede ayudar a explicar la creciente polarización sufrida por el electorado después del gobierno de Ibáñez. ¿Por qué una vez muerto Ibáñez, el ibañismo (en contraposición, por ejemplo, al peronismo) dejó de ser una corriente significativa? ¿Qué tuvo que ocurrir para que los chilenos optaran por los partidos políticos "consolidados", como la Democracia Cristiana, el Partido Nacional o las agrupaciones de la Unidad Popular? ¿A qué se debe que la "laxitud" — ¿pragmatismo?_- de los independientes como Ibáñez hubiera sucumbido ante las posturas rotundas y definitivas de los años inmediatamente anteriores a 1973? Por último, ¿cuál es el lugar del ibañismo en el contexto latinoamericano?

Sin duda, esta obra invita a reflexionar sobre la naturaleza del populismo y su constante aparición y reaparición en el ámbito latinoamericano ${ }^{4}$. El autor hace mención a este punto en su introducción, pero, ya por falta de tiempo o de espacio, lamentablemente no le dedica mayor atención. Habría sido interesante saber la opinión de Fernández respecto, por ejemplo, al "neo populismo" de Hugo Chávez, Lula da Silva o Rafael Correa, como asimismo haber extendido las características de Ibáñez hacia otros personajes de la historia de Chile. René Millar, en un conciso pero lúcido prólogo,

${ }^{4}$ Según Alan Knigh, op. cit., p. 223, "el populismo parece ser un 'moribundo eterno'. Declarado muerto y sepultado sin sollozos, con la estocada en el pecho vuelve, como los muertos vivos de la política latinoamericana, a rondar el mundo de los sentidos, impertérrito ante el radiante amanecer de la democracia y el neoliberalismo". El original en inglés dice: "populism seems to be an 'unconscionably long time dying'. Pronounced dead, buried, unlamented, with a stake through the heart, populism returns, like the living dead of Latin American politics, to haunt the sentient world, undeterred by the bright dawn of democracy and neo-liberalism". 
se refiere a algunas de las características del General perfectamente atribuibles a Jorge Alessandri, hijo del enemigo máximo de Ibáñez pero tan antipartidista como él. ¿Podrían encontrarse otros casos en la historia de Chile similares al de Jorge Alessandri? Esperamos que, más temprano que tarde, el autor vuelva sobre este tema.

Palabras clave: Historia política de Chile; Carlos Ibáñez del Campo; ibañismo; populismo.

Recibido: diciembre 2008. Aceptado: enero 2009. 